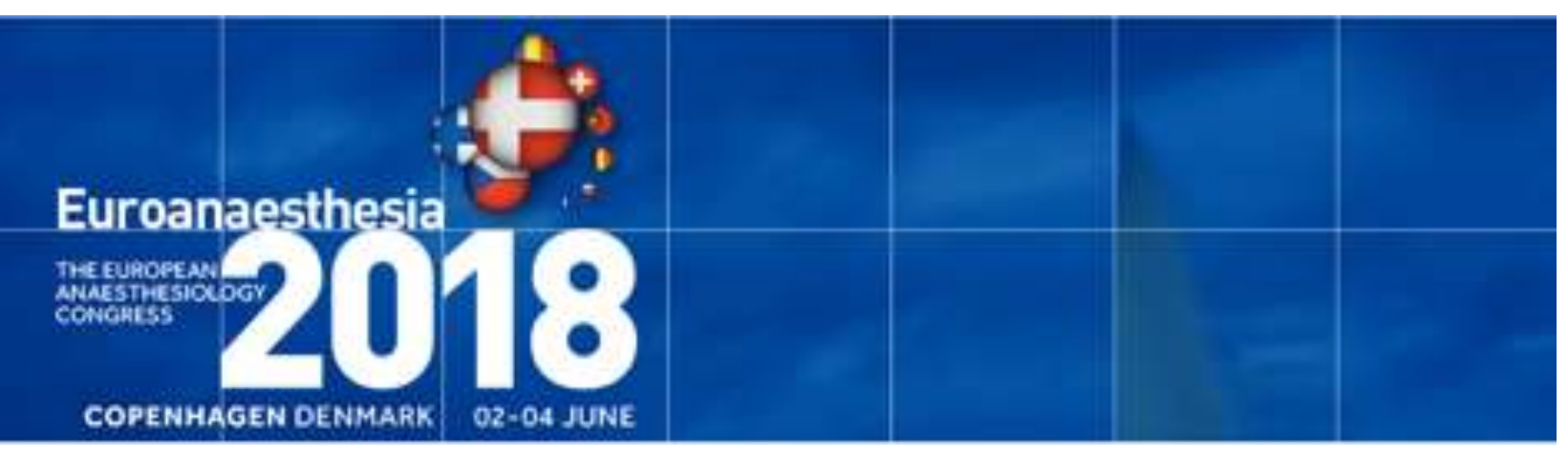

\title{
CRITICAL MANAGEMENT OF THE PREGNANT PATIENT WITH AN ATYPIC HEMOLYTIC UREMIC SYNDROME
}

\section{Dra. Sánchez, Ma Elena; Dra. Carbonell, Nuria; Dr. Oglio, Juan Pablo; Dra. Raynard, Mireia;} Dra. Fernández, Marisol; Dr. Miranda, Alejandro

Hospital Universitario Dexeus (Grupo Quirón-Salud)

\section{Background:}

Up to $86 \%$ of patients with hemolytic uremic syndrome (HUS) secondary to pregnancy have mutations in the alternative pathway of complement, the underlying disease being an atypical HUS (aHUS) ${ }^{2}$. It is an ultra-rare disease that usually evolves to a terminal chronic renal failure, which makes it an easily underdiagnosed and potentially lethal pathology ${ }^{1}$.

\section{Case Report:}

Pregnant woman of $19+4$ weeks that consults in the Emergency Room for right lumbar pain, micturition syndrome and fever. The sediment is compatible with urinary tract infection, which is corroborated with abdominal ultrasounds. E. coli is isolated, both in blood and urine cultures, so it is admitted under suspicion of acute obstructive pyelonephritis. The physical state and the analytical tests corroborate severe sepsis. The patient is transferred to the intensive unit care with perfusion of Noradrenaline at $0.8 \mathrm{mg} / \mathrm{h}$.

It appears hemolytic intravascular anemia with negative Coombs, worsening thrombocytopenia and renal function. The schistocyte visualization, with negative Adamts13, confirms aHUS. Immunoglobulin $1 \mathrm{~g} / \mathrm{kg}$ is administered, achieving normalization in 14 days. In week $38+6$, cesarean section is scheduled, which takes place without incidents, under intradural anesthesia.

\section{Discussion:}

aHUS is an entity that is defined by the clinical triad of non-autoimmune hemolytic anemia, thrombocytopenia and acute renal failure. The choice of the anesthetic technique in pregnant women with thrombocytopenia is multifactorial. The number of circulating platelets and their function determines the safety of regional anesthesia ${ }^{3}$. Subarachnoid blockade with small caliber needles makes it a much less traumatic technique and a good choice for patients with aHUS. General anesthesia will be a good alternative in caesarean sections if there is evidence of bleeding or coagulation alteration.
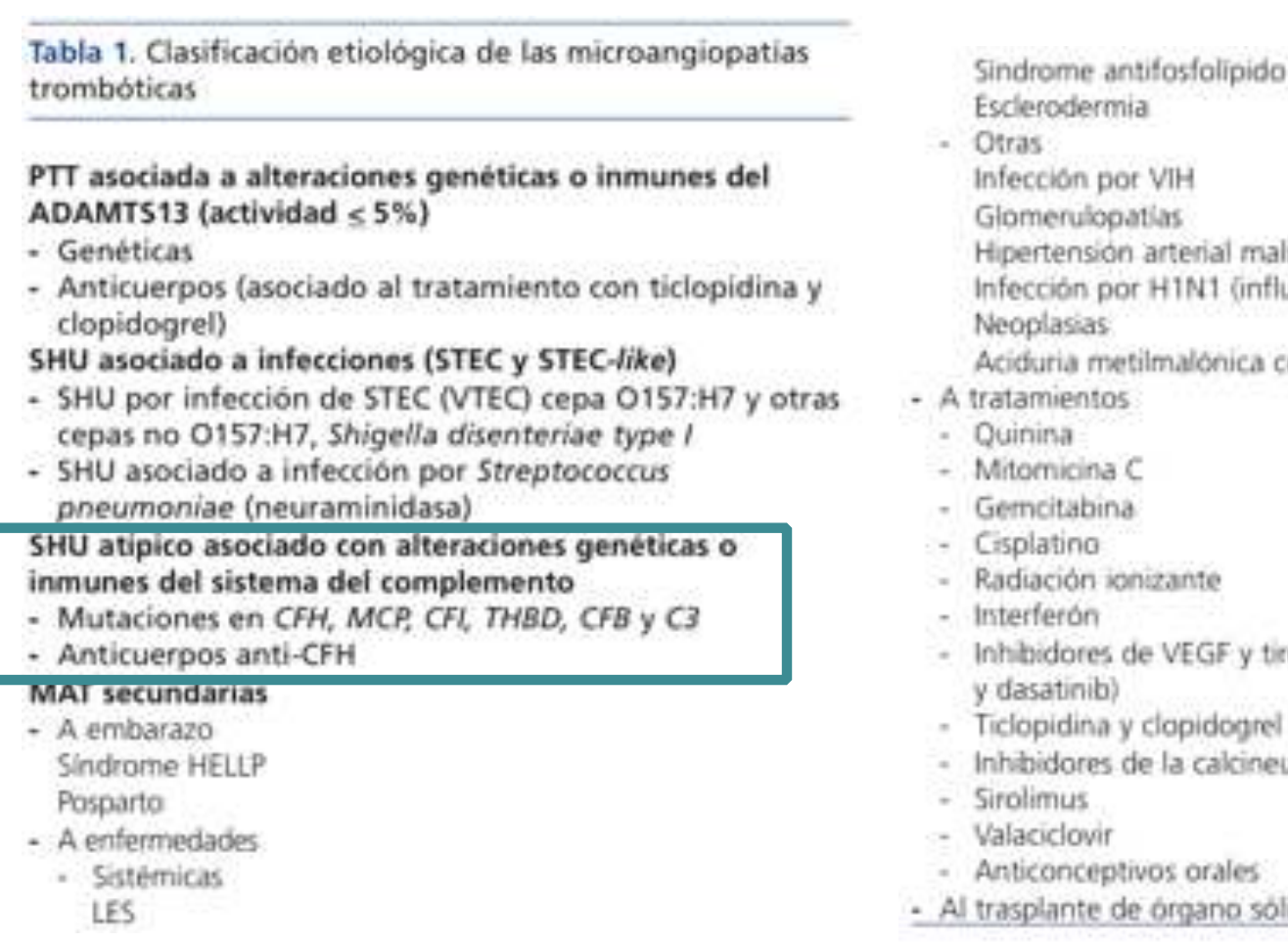
Otras Glomerubopation Hipertension arterial maligna Neoplasias aciduria metilmalonica con homoxistinuri A tratamientos Quinina Mitominina Gemcitabin Gisplatina Interferón Whibidores de VEGF y trosina quinasa (sunitinib, imatinity y dasatinib dopidina y clopidogrel (a) Sirolimus Anticonceptivos orales Al trasplante de ómano solida o de médula ósea

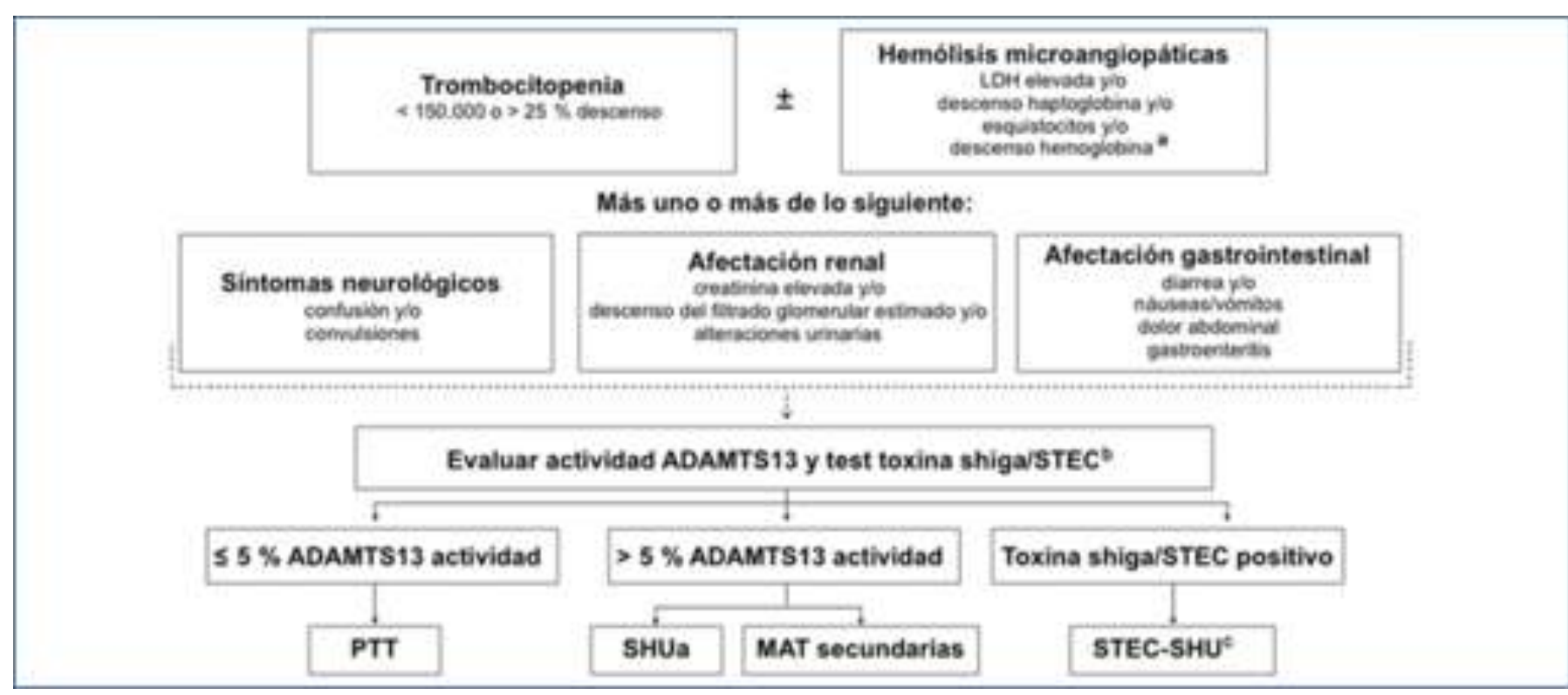

\section{VAGINAL BIRTH:}

- If platelet count $>70.000$ and there is no other contraindication: Locoregional anesthesia

- If platelet count $<70.000$ and / or contraindication of neuraxial block: Opioids systemically

\section{CAESAREAN SECTION:}

- If platelet count $>70.000$ and there is no other contraindication: Locoregional anesthesia

- If platelet count < 70.000 and / or contraindication for neuraxial block: General anesthesia

\section{Key Point:}

In every pregnant woman affected by HUS, a thorough preanesthetic evaluation must be carried out, weighing the advantages and disadvantages of the different anesthetic techniques. The safest anesthetic management depends on a deep knowledge of the disease and its implications. 